\title{
Developing Empathy for People with Bipolar Disorder Through Interactive Performance
}

\author{
Kyungho Lee \\ Illinois Informatics Institute \\ University of Illinois at Urbana-Champaign \\ 1205 W. Clark NCSA Building, Urbana, IL, USA \\ klee141@illinois.edu
}

\begin{abstract}
Misplaced Euphoria is an interactive performance that consists of expressive movement, expressive gesture recognition, and interactive visualization that aims to develop empathy for people with bipolar disorder. This project explored the characteristics of those with bipolar disorder through the mediums of dance and interactive visualization driven by machine learning. To develop an interactive performance, we conducted an interview with a person to gather information and use the in- sights collected from the interview processes as a base for our creative process. We used a support vector machine (SVM) to recognise the dancers expression and gestures recognition results were visualised through the Rutt-Etra algorithm on a real-time basis.
\end{abstract}

Interactive performance. Bipolar disorder. Expressive gesture. Kinesthetic empathy. Machine learning.

\section{INTRODUCTION}

Violent. Unpredictable. Dangerous. Crazy. Distrustful. Outcast. These terms are just some examples of the stigmas that exist against people with mental illness for which we hope to raise awareness, because misunderstanding and prejudgment may hurt the people who are suffering from bipolar disorder even further.

Scientifically speaking, bipolar disorder-also known as manic-depressive illness-is a brain disorder that causes unusual and unpredictable shifts in mood, energy, activity levels, and the ability to carry out daily tasks. It has been reported that bipolar disorder symptoms can result in damaged relationships, poor job or school performance, and even suicide. However, it is also believed that bipolar disorder can be treated and that people with this illness can lead full, ordinary, and productive lives.

Nevertheless, it is said that the biggest obstacles in bipolar disorder treatment are more societal than scientific. It is true that some people to be treated or get the right treatment as it is chronic-not only because it does not exist but also because they are afraid of the judgment and misconception that so often comes with accepting a mental health diagnosis and seeking treatment for it. Some might refer to this stigma. Nowadays, this is considered to be discrimination and it is this current perspective that inspires us to initiate this project.

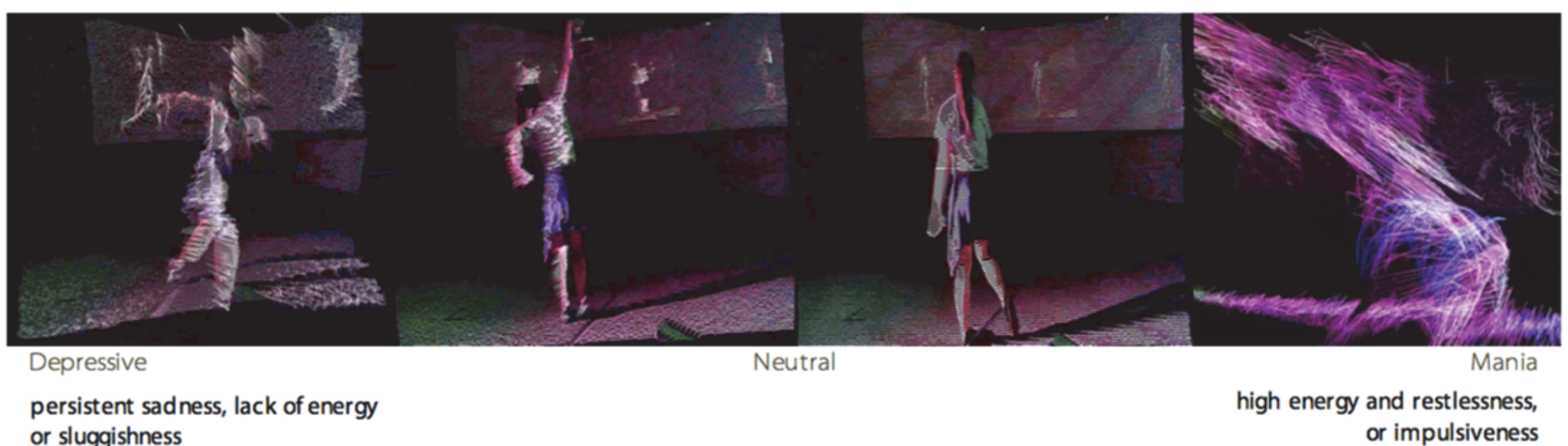

Figure 1: Interactive performance to develop kinaesthetic empathy driven by machine learning. 

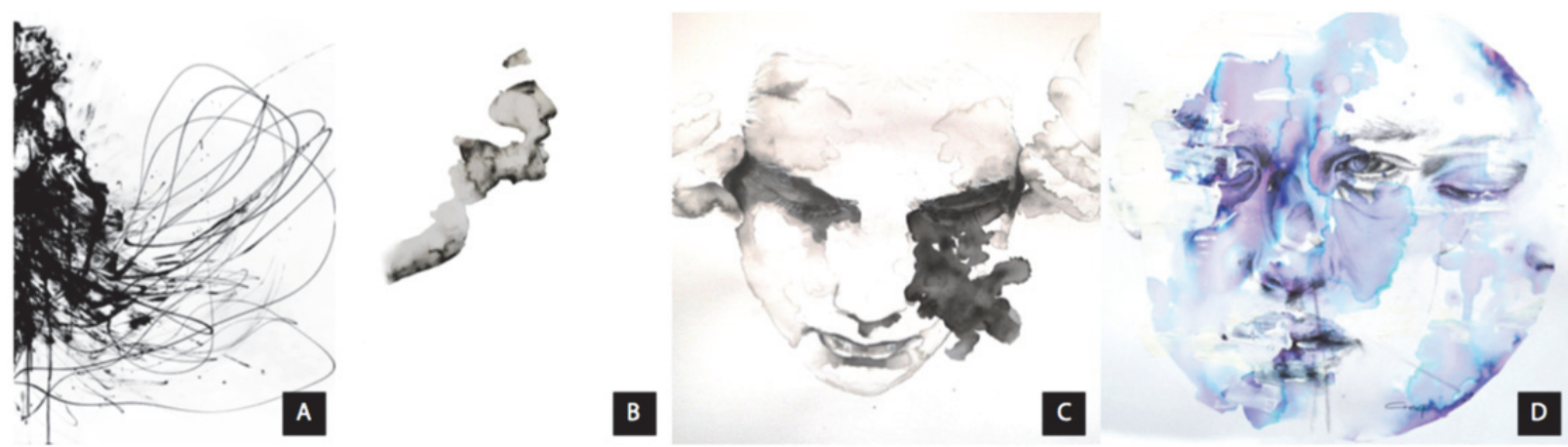

Figure 2: (A) Original sketch drawn by an interviewee, $(B),(C)$ : Sketches drawn by an artist inspired by what it feels like to be depressive (D) Sketches drawn by an artist to depict the manic mood.

To develop empathy for people with bipolar disorder and open a new dialog for them, we present an interactive dance performance called Misplaced Euphoria. This project explored the characteristics of those with bipolar disorder through the mediums of dance and of emerging technology that recognises characteristic movements, representing it in the form of an interactive visualization using machine learning.

This interactive performance aimed to provide an opportunity to take the time to think about and discuss the way we look at these issues through an aesthetic experience, hoping that the audience may be able to experience a form of "inner mimesis," where they felt as if they were suffering from bipolar disorder while they were observing the interactive visualization.

\section{DESIGN FOR EMPATHY}

It is suggested that empathy is defined as an innate human ability to understand and share the feelings of another Davis (2018) noted. According to Coulton et al. (2014), empathy is an emotional capability that has often been overlooked within the design of interactive systems. This phenomenon may be problematic for some areas, as empathy is considered to be, and functions as, one of the foundational human emotions, representing essential components that define the human condition to embrace others, especially for people who have different diagnoses, such as bipolar disorder, autism spectrum disorder, or AttentionDeficit/Hyperactivity Disorder (ADHD).

If empathy is such an important aspect of humanity, then it should be considered in the design of digital communication systems and interactive works. For this reason, designing for empathy can be thought of as an important matter even though the topic has not received much attention thus far.

From this perspective, Belman and Flanagan (2010) suggested that there is rich literature related to the topics of how empathy is experienced and how empathy influences one's attitudes and behaviour. There is also a lack of discussions and practices on how the $\mathrm{HCl}$ research and design should incorporate empathy in order to convey it to the users appropriately (Boehner et al. 2005; Gaver 2009). That is why we attempted to design a dance performance using interactive technology that can incorporate empathetic feelings and call for behavioural changes in the audience through employing the theory of kinaesthetic empathy.

As Belman and Flanagan (2010) further noted, empathy may help people improve attitudes and motivate prosocial behaviour (voluntary behaviour intended to benefit others). Based on this claim, we hope our performance could promote empathy in people who are not aware of such diseases and help explain to them what it feels like. It is also our hope that it would help to increase the happiness and wellbeing of those who are both directly and indirectly related to the disease by improving the coherence and "spirit" of the community through the performance.

\section{INTERVIEW}

To collect information that may be used as a base for our creative process, we conducted a series of interviews with a person with bipolar disorder. Our interviewee was a 40-year-old man, who has a BA/MA in journalism and was diagnosed with bipolar disorder when he was in high school. We transcribed each word carefully when our interviewee described any changes in his emotions and their relationship to the objects and events that occurred around him. For example, he described the feeling of living with bipolar disorder as follows: "when you're bipolar, well, it feels like there is a giant pendulum in your head swinging between being extremely self-critical and awkward and suddenly having an immense amount of confidence then it goes away in a second. It's like turning some switch on and off in your head continuously." He also provided us with a sketch to illustrate what it feels like to have bipolar disorder as shown in Figure 2. 


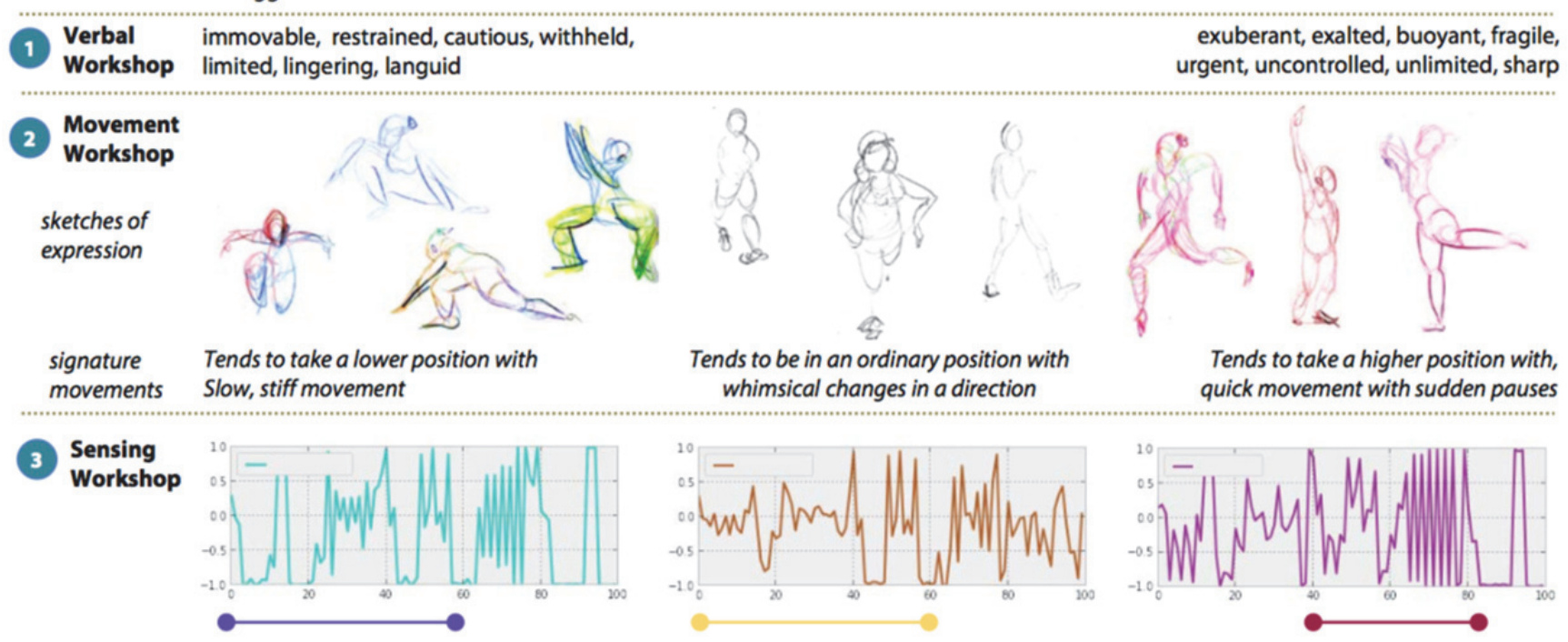

Figure 3: Diagram shows how we develop an interactive performance through multiple workshops.

\section{DESIGN PROCESS}

Based on the conceptual design, we went through multiple workshops-a verbal workshop, a movement workshop, and a sensing workshop-as illustrated in Figure 3. We first conducted a verbal workshop in which both the designer and the dancer tried to build a conceptual consensus about the symptoms. We verbalised the feelings and emotions using a visual thesaurus. For example, we chose some words that were collected during the interview and then used them as a starting point. We kept finding additional words that may also represent the meaning of the collected words in a more precise and accurate manner so that we could build strong consensus.

Then, the dancer began to create expressive gestures to represent the feelings and inner states that were found in the verbal workshop. While the dancer focused on the movement, the designer had great visual inspirations from the process and created some sketches to convey the dancer's intention, as well as feelings, effectively. In this phase, the dancer was immersed into creating expressive gesture sequences that might evoke kinaesthetic empathy, allowing herself to resonate and let her body be moved by the experience of interviewees.

Two different perspectives, mirroring and resonating, were adopted and explored in this process - the first is externally oriented, while the second is more inner-directed. These perspectives were the main tools used by the dancer to understand the interviewee's traumatic experiences and to represent their significance through expressive body movements that would eventually be exaggerated in the visualization. We believed that dancers are able to relate to both inner and outer worlds, which develop empathy enabling intimacy and human closeness through movement, perception, understanding, and intervening. During this stage, various visual metaphors were created and applied for the projection.

The sensing workshop was conducted last. During the workshop, two 6-DOF accelerometers, attached to the dancer's right and left wrists, were used to capture her movement. We collected four hours of expressive movement datasets that represent feelings and inner states. We used an SVM with a Gaussian kernel function to train and recognise the dancer's expression. Since the data have two classes, labelled as mania and depressive, we considered an SVM to be a good fit for achieving our goal. The results were visualised through the Rutt-Etra algorithm Furlong, L. (1983) on a realtime basis as shown in Figure 1.

\section{EXPRESSIVE GESTURE RECOGNITION}

An SVM is considered to be very competitive within the existing classification methods in many areas, and it has drawn the researcher's attention due to its elegant mathematical reasoning for selecting the optimal hyperplane that maximises the margin between two classes. Since our gesture dataset was not linearly separable, a kernel function-in 
this case, a radial basis function (Gaussian)-is used to transform the data into a new space so we can separate it in a higher dimension.

To build the expressive gesture recognition model that recognises two moods, we captured the dancer's expressive movement sequences. For example, we collected information generated by two accelerometers while the dancer expressed the depressive side of the disorder for the first two hours and the mania side for the remainder of the time. We labelled each sequence differently so that we could perform a binary classification supported by the SVM.

We trained the model with a feature generated by a sliding window approach. We applied the leaveone-out cross-validation, where one single sequence is selected for a test at a time, using the rest as training examples. Subsequently, we went through an optimization process. To estimate the best hyperparameters, we carried out a grid search in the space of parameter $C$ and $Y$, where $C$ represents the weight of error penalty and $Y$ determines the width of the RBF kernel. An optimised SVM classifier can be selected by the set of two parameters of $(C, y)$, which maximises the cross-validation rate in the space of search, which, in turn, increases the accuracy of the results. This result suggested that the model needs to have 15 frames as a window to predict the incoming expressive sequences appropriately. Any notable latency/time-delayed effects that might distract from the dancer's performance were observed in the pilot test, as we used a large amount of movingaverage between the state transitions.

\section{CONCLUSION}

People living with bipolar disorder keep emphasizing that the biggest obstacles they face in their everyday lives are more societal than scientific. In many cases, they are fighting and struggling with prejudice and misconception that prevails through media. While our interactive performance is developed as a work in progress, this research and practice are intended to stimulate debate around designing for empathy, particularly for those who suffer from bipolar disorder.
To develop empathy for people with bipolar disorder this project was initiated and developed over time. In the design process, the designer and the dancer explored the characteristics of those with bipolar disorder through the mediums of dance and interactive visualization driven by machine learning.

The intent is that this type of performance will have a positive impact on the psyche of the audience through the theory of kinaesthetic empathy as a vehicle for developing empathy for people who suffered from bipolar disorder. We hope the audience can thus have the chance to experience the inner life of people with bipolar disorder and to catch a glimpse of it in order to understand what it feels like, thus avoiding any further misunderstanding and prejudgment that may hurt those who suffer.

\section{REFERENCES}

Bearden, C. E., Hoffman, K. M. and Cannon, T. D. (2001) The neuropsychology and neuroanatomy of bipolar affective disorder: a critical review. Bipolar Disorders 3(3): 106-150.

Berrol, C. F. (2006) Neuroscience meets dance/movement therapy: Mirror neurons, the therapeutic process and empathy, The Arts in Psychotherapy, 33(4): 302-315.

Daly, A. (1992) Dance History and Feminist Theory: Re-considering Isadora Duncan and the male gaze. Gender in performance: The presentation of difference in the performing arts, pp.239-59.

Fairclough, S.; Robinson, R. K.; Nichols, D. L.; and Cousley, S. 2013. In Sickness and in Health: Implications for Employers when Bipolar Disorders are Protected Disabilities. Employee Responsibilities and Rights Journal, 25(4):277292.

Furlong, L. (1983) Notes toward a history of imageprocessed video: Eric Siegel, Stephen Beck, Dan Sandin, Steve Rutt, Bill and Louise Etra. Afterimage, 11(1).

Koch, S. C., Fuchs, T., Summa, M., and Muller, C. (2012) Body Memory, Metaphor and Movement. John Benjamins Publishing. 